\title{
When an emerging disease becomes endemic
}

Graham F. Medley* and Anna Vassall

Department of Global Health and Development, London School of Hygiene and Tropical Medicine.

*Corresponding author. E-mail: Graham.Medley@lshtm.ac.uk

\begin{abstract}
Epidemics, such as HIV in the early 1980's and Ebola in 2014, inspire decisive government investment and action, and individual and societal concern, sometimes bordering on panic. In contrast, endemic diseases, such as HIV in 2017 and tuberculosis, struggle to maintain the same attention. For many, the paradox is that endemic disease, in its totality, continues to impose a far higher public health burden than epidemic disease. By and large, the swift political response to epidemics has resulted in success. It has proven possible to eradicate epidemic diseases, often without the availability of vaccines and other biomedical technologies. In recent times, only HIV has made the transition from epidemic to endemic, but diseases that have existed for centuries continue to cause most of the infectious disease burden.
\end{abstract}




\section{Main text}

Characterization of a disease as either epidemic or endemic is commonly understood epidemiologically, but we argue that the social framing of a disease as 'epidemic' or 'endemic' is equally important. For livestock, the relationship between disease phase and social response is made more explicit: diseases are defined as "exotic", and thence acquire a legal status requiring government action to eliminate them. Other endemic or livestock "production diseases" are permitted to remain endemic (1). By contrast, in human health, disease status and responses are rarely legally determined. As with animal diseases, human diseases acquire a social status, primarily based on their perceived risk, that determines their acceptability and the level of intervention deemed appropriate. In this exploration of the characteristics of epidemic and endemic infectious disease, we highlight the pivotal role of risk and risk perception in explaining individual and societal responses to diseases in different phases of their establishment in populations. The heterogeneity of risk across populations is one of the key aspects we consider. We argue that individual and societal risks both determine, and are determined by, the classification of a disease as an epidemic or endemic. Thus, the classification of disease as 'epidemic' or 'endemic' reflects both biological and social phenomena.

\section{The public response}

Responses to 'epidemics' tend to be public, seemingly without resource constraints, and often combine the efforts of national and global public health institutions. For example, HIV provoked over a ten-fold rise in development funding for health in a decade, and the forming of new public institutions such as UNAIDs (2). As diseases become 'endemic', they become increasingly tolerated, and the locus of responsibility may shift to the individual. Rather than public authorities actively detecting cases and subsiding risk protection, people may be increasingly encouraged to pay for the means to manage their own risk and seek care. Likewise, the focus of any global response may move away from direct provision of services by international agencies, to other forms of intervention, 
such as building national capacity more generally, supported by domestic financing (3).

For many, the primacy of the national and global response to epidemics appears an "over-reaction", as resources are pulled in across government departments to rapidly control and limit the outbreak. Responses may thus be perceived as being at the detriment to other health priorities, such as routine vaccination. Epidemics may provoke broad multi-sectoral responses led by the political executive, mass information campaigns and military mobilization. Epidemics may also mobilize substantial public investment in vaccines or the development of treatments: the UK government is estimated to have spent 1.2 billion pounds sterling on the swine flu epidemic (4), and licensing arrangements for diagnostics, medicines and vaccines in emergencies are relaxed (5). The rationale for the political imperative is two-fold. First, epidemics can most (cost)effectively be controlled when the number of cases is very small, and thus even an ill-informed rapid response may be more beneficial and efficient than a cautious informed one (6). Second, there is a (often highly uncertain) risk of catastrophic impact: epidemics have destroyed civilizations. Politicians therefore need to weigh investment against highly uncertain, but potentially devastating social, health and economic consequences. The "public health paradox" ensures that if the epidemic is successfully controlled, then it is highly likely that the eventual impact of the epidemic disease will have been less than the opportunity cost of the resources allocated to it from other health areas. The experience of 1918 still serves to remind us, that had we not responded to H1N1 as we did, it could have been much, much worse.

At some point, the socio-political response to an emerging disease starts to change. Investment in the disease may become institutionalized in the health sector - with those who recognize the importance of broader social drivers and impact of infectious disease struggling to mobilize other sectors. For example, despite the long acknowledged association between poverty and tuberculosis (TB) (7) combined health and social intervention remains a rarity, and are only now being pioneered in countries such as South Africa where TB has embedded 
itself as the largest killer. Financing may stabilize or diminish, in part as other disease areas or the health system recuperates from any temporary loss of funding caused by the epidemic (2). Resourcing the response becomes increasingly based on known risks and benefits that can be more clearly compared with alternative investments in the health sector or beyond, articulated in 'investment cases' for specific diseases (8). Health insurance organizations, which at this stage are able to predict risk, may start to cover any response in their insurance benefits (and premiums). The disease itself takes on an "identity", and interest groups form out of the populations where the disease is becoming 'endemic' often advocating for attention and action. The disease now must compete for attention and resources with other endemic diseases, even if the benefits of disease control still clearly outweigh the costs (8).

\section{The private response}

The public responses to epidemic and endemic diseases mirror those of individuals. At a personal level, epidemics may inspire panic largely because the risk of acquisition of infection is impossible to gauge, and treatments are limited. As individuals act to avoid (often highly unknown) risks, they are willing to behave in ways that may have substantial social and economic costs and consequences. People may avoid work, take children out of school, and flee or

minimize travel (9). This reaction and its consequential costs are often not borne evenly across populations. The ability of an individual to act is constrained by economic and social circumstances, and thus even at an early stage, epidemics start to impact different groups in society differentially, as has been documented for the Ebola virus epidemic in Liberia (10). Stigmatization of those perceived to be at greatest risk of infection and transmission is common.

As epidemics transition into endemic disease, people develop a perception that they understand the risks of infection, giving them a sense of control. Generally, people cope with risk by adjusting behavior and mitigating the consequences, often to the point at which any new behaviors become a tolerated part of life. The move of the locus of responsibility from government to individual can then 
be enabled by a belief that individuals can now make informed choices, even where those individuals are highly constrained by their circumstances. For example, new forms of funding the means of risk protection may emerge, such as the social marketing of condoms or malaria bed-nets, ultimately sharing the financial burden of the disease even among the very poor. As a disease becomes endemic, governments generally still provide some funding for treatment, but in the context of resource scarcity, governments may fail to fund universal access, leaving many to access private care, even where treatment may prevent others from being infected, such as the case of TB or HIV. For many interest groups this is an "under-reaction", compared with the epidemic response. Even when the disease has settled into populations, these groups may continue to sustain the political imperative associated with epidemics (11); calling for urgent action to end the disease, unfortunately often with limited success.

\section{The determinants of risk}

The risk and perception of risk that drives the societal response to disease is a combination of the probability of infection coupled with the consequences of infection - the widespread fear associated with epidemics, is often driven by the lack of effective treatment. In 2017, most of us would prefer a diagnosis of HIV to one of Ebola virus, but the distinction would have been less clear prior to 1995 when less effective drugs were available. Epidemic diseases typically have higher mortality and morbidity than endemic diseases, owing to lack of clinical experience and knowledge, as well as innate pathogenicity. Over time, effective prevention and treatment interventions emerge. However, although improved treatment is clearly a good thing, and reduces the risk of catastrophic loss of individual health, the reduction in risk may also provoke a decline in political interest, initiating a more endemic style response. Where anti-microbial resistance emerges, reducing the treatability of endemic diseases such as TB, fear may return and one again inspire a more 'epidemic' style response.

Paradoxically, the amount of public funding needed to respond to infectious diseases may increase during a transition from epidemic to endemic, as those 
organizations which made the initial investments in medical technologies to prevent, diagnose and treat the disease attempt to recoup their investment. The resource estimates for HIV were moderate initially, but as effective treatment emerged, increased substantially (8). Likewise, the availability of more effective treatment may increase costs to households, as the disease becomes chronic rather than acute, leaving households having to deal with the costs of long-term illness, and of accessing care. For some population groups, unable to afford the costs of accessing treatment (7), this can worsen the endemic and entrenched nature of the disease, by reinforcing cycles between risk and poverty; potentially exacerbating initial differentiation of risks that emerged in the epidemic stage.

If diseases become entrenched in certain population groups, and without substantial investment and political will, endemic diseases then may become very expensive and difficult to control, eliminate and eradicate. Immunization is generally seen as essential for eradication campaigns, although Guinea worm is likely to be the first infection eradicated without recourse to immunization. Global-scale elimination and eradication of tuberculosis, malaria and (now) HIV appear to be a long way off, but the biomedical tools we have for these diseases are effective, plentiful, and relatively low cost, and offer huge potential to reduce the disease burden substantially. These tools are substantially better than those available for severe acute respiratory syndrome (SARS), Middle East respiratory syndrome (MERS) and Ebola, all of which have been successfully eliminated. The difference is in the political willingness to provide the necessary funding at the scale required, reflecting an intrinsic societal acceptance that populations should tolerate risk of infection in a way that they do for other health interventions.

Yet, individuals are not at equivalent risk of acquiring or transmitting infection in epidemic or endemic phases (12). As epidemics proceed, infection becomes increasingly associated with socially defined groups, which are at higher risk of infection and usually a small proportion of the population. However, the behaviors that make the risk of infection for some groups higher are not exclusive to those groups, and as the disease becomes endemic, the importance of the high-risk groups that dominated in epidemics is reduced. For example, at 
the start of the HIV epidemic, individuals with the highest rates of sexual partner change are more likely to be infected, and transmit at a higher rate: they are core to the epidemic's progression. As HIV becomes more endemic, individuals with the same behavior remains at high individual risk of infection with HIV, but the majority of transmission might come from individuals with lower rates of sexual partner change. This is for two reasons. First, the number of infected individuals with lower rates of sexual partner change is much greater than those with high rates of sexual partner change. Second, the status of the partners is different people at high risk are at high risk because a high proportion of their partners are already infected, whereas people with lower rates of change are more likely to have partners who can be infected. Consequently, as infection disseminates through populations, the risk of acquisition and the rate of transmission become less well correlated.

Therefore, as epidemic disease transitions into endemic disease, even though it becomes entrenched in specific groups, it also may increasingly move towards Rose's "prevention paradox": that is small, common risks are responsible for more disease than large, rare risks (13). Additionally, individuals might get infected in one group and transmit to another. For example, older male partners might transmit HIV to adolescent girls and young women, who then infect their male peers (14). It is no longer the case that funding can be targeted intensively at small high-risk groups. Groups with the highest risk of infection become increasingly decoupled from those with the highest risk of dissemination. This complicates question of allocation of scarce resources for endemic diseases: should they be allocated to those most likely to transmit or those most likely to be infected?

In the early phases of epidemics, populations at risk are usually considered constant and births and deaths and life-course of behavior, which may result in transitions between risk groups, are ignored. For example, commercial sex workers are at high risk of HIV infection, but there is relatively little consideration given to their risks prior to entering this category, or after they leave. In the transition to endemicity, social and economic transitions continue to 
be critical to understanding how infection is disseminated in a population, and the dynamics of the individual risks of infection and transmission. Actions taken by individuals to address their poverty could lead to the spread of the disease, for example, the movement of female sex workers around festivals in India, or miners in South Africa with tuberculosis returning home. Migrating populations, such as refugees, may also have poor access to risk protection, as they often are the groups least covered by health and social insurance.

The evolving risk of infection during endemicity can therefore be most easily understood and predicted in terms of structural drivers of risk, i.e. the economic, political, social factors that determine the size of these groups, and individuals' exposure to infection and ability to access prevention and treatment services (15). Over time, inequalities in risk can become entrenched, reflecting social structures and constraints, and, as with other form of inequities become, essentially, tolerated. In the UK, it is accepted that TB is associated with homelessness and recent immigration, and HIV is associated with men who have sex with men. Disease often continues to be endemic in specific population groups despite the availability of interventions to reduce transmission (i.e. condoms, microbicides), and effective treatments, reflecting the social and economic constraints to access and use of means of prevention and treatment services.

\section{Conclusion}

We have argued that in the wider public arena epidemics and endemics are distinguished by the individual and social perceptions and tolerance of risk. At a public level, we have the endemic diseases we have because widespread multisectoral action, eradication or elimination are not sufficiently politically appealing, despite the fact that investment is often cost-effective. The contained public response, and the concurrent shift of responsibility to individuals to protect themselves from risk, means that endemic disease embeds itself further, as those at risk are often the very same people who do not have the private resources to avoid risk or access treatment. The endemic diseases we have are 
those that have found a "niche" in social geography, and often they reinforce that niche. The labels 'endemic', 'intractable' and 'non-eradicable' are self-fulfilling descriptions of settled endemicity.

Actions to address the social and structural determinants of infectious disease are increasingly being embedded in the response to both epidemic and endemic disease. The better the infrastructure and systems of preparedness and rapid responses to epidemics, the better we avert the unnecessary expenditures and costs associated with rapid action in the face of uncertain, high-risk consequences. Encouragingly, the use of approaches rooted in anthropology and the social sciences are now beginning to be integrated in the infrastructure of early epidemic responses, and may improve efficiency of the response and prevent the emergence of endemicity in specific population groups (16). For diseases that are 'endemic', there is increasing policy recognition that a combined social and biomedical response is central to any strategy for elimination $(17,18)$. Examples of the success of combined social and biomedical intervention, such as the scale-up of community based HIV prevention in India, are encouraging (19). However, there is still much to be done before social intervention is a commonplace component of infectious disease responses. Acknowledging that epidemics and endemics are intrinsically socially as well as epidemiologically defined is a critical step forward in developing the comprehensive response that is required to address the complex challenge of infectious disease elimination. 


\section{References}

1. D. Carslake et al., Endemic cattle diseases: comparative epidemiology and governance. Phil. Trans. R. Soc. B. 366, 1975-1986 (2011).

2. Global Burden of Disease Health Financing Collaborator Network.. Evolution and patterns of global health financing 1995-2014: development assistance for health, and government, prepaid private, and out-of-pocket health spending in 184 countries. Lancet. 2017 May 20;389(10083):1981-2004.

3. Vogus A, Graff K. PEPFAR Transitions to Country Ownership: Review of Past Donor Transitions and Application of Lessons Learned to the Eastern Caribbean. Glob Health Sci Pract. 2015 Jun 17;3(2):274-86.

4. https://www.gov.uk/government/publications/report-of-the-swine-flucritical-care-clinical-group-and-key-learning-points-for-future-surgeplanning'. Accessed 11/06/2017

5. WHO (2015) Assessing new medical products in health emergencies: the EUAL procedures. WHO Drug Information Vol. 29, No. 3, 2015. Available at:http://www.who.int/medicines/publications/druginformation/issues /WHO DI 29-3.pdf?ua=1, accessed 13 June 2017

6. M. J. Keeling, M. E. J. Woolhouse, R. M. May, G. Davies, B. T. Grenfell, Modelling vaccination strategies against foot-and-mouth disease. Nature. 421, 136-142 (2003).

7. Foster N, Vassall A, Cleary S, Cunnama L, Churchyard G, Sinanovic E. The economic burden of TB diagnosis and treatment in South Africa. Soc Sci Med. 2015 Apr;130:42-50.

8. Schwartländer B, Stover J, Hallett T, Atun R, Avila C, Gouws E, Bartos M, Ghys PD, Opuni M, Barr D, Alsallaq R, Bollinger L, de Freitas M, Garnett G, Holmes C, Legins K, Pillay Y, Stanciole AE, McClure C, Hirnschall G, Laga M, Padian N; Investment Framework Study Group.. Towards an improved investment approach for an effective response to HIV/AIDS. Lancet. 2011 Jun 11;377(9782):2031-41.

9. M. R. Keogh-Brown, R. D. Smith, J. W. Edmunds, P. Beutels, The macroeconomic impact of pandemic influenza: estimates from models of the United Kingdom, France, Belgium and The Netherlands. Eur J Health Econ. 11, 543-554 (2009).

10. Kastelic, Kristen Himelein; Kastelic, Jonathan G.. 2015. The socio-economic impacts of Ebola in Liberia : results from a high frequency cell phone survey 
round five. Washington, D.C. : World Bank Group. http://documents.worldbank.org/curated/en/162381468179333776/T he-socio-economic-impacts-of-Ebola-in-Liberia-results-from-a-highfrequency-cell-phone-survey-round-five

11. D. W. Brinkerhoff, Building political will for HIV response: an operational model and strategy options. Int J Health Plann Mgmt. 31, 470-487 (2015).

12. M. S. Y. Lau et al., Spatial and temporal dynamics of superspreading events in the 2014-2015 West Africa Ebola epidemic. PNAS. 114, 2337-2342 (2017).

13. G. Rose, Sick Individuals and Sick Populations. Int. J. Epidemiol. 14, 32-38 (1985).

14. Schaeffer R et al. Age-disparate relationships and HIV incidence in adolescent girls and young women: evidence from a general-population cohort in Zimbabwe. AIDS, online edition, 2017. DOI: 10.1097/QAD.0000000000001506

15. J. O. Parkhurst, HIV prevention, structural change and social values: the need for an explicit normative approach. - PubMed - NCBI. JIAS. 15, 1-10 (2012).

16. A. Wilkinson, M. Parker, F. Martineau, M. Leach, Engaging "communities": anthropological insights from the West African Ebola epidemic. Phil. Trans. R. Soc. B. 372, 20160305 (2017).

17. E. Michael, S. Madon, Socio-ecological dynamics and challenges to the governance of Neglected Tropical Disease control. Infectious Diseases of Poverty 2017 6:1. 6, 35 (2017).

18. K. F. Ortblad, J. A. Salomon, T. Bärnighausen, R. Atun, Stopping tuberculosis: a biosocial model for sustainable development. The Lancet. 386, 2354-2362 (2015).

19. M. R. Pickles et al., Assessment of the population-level effectiveness of the Avahan HIV-prevention programme in South India: a preplanned, causal- 
pathway-based modelling analysis. Lancet Global Health. 1, e289-e299 (2013). 